\title{
Evaluación del estudio de contactos de tuberculosis. Servicio de Salud Metropolitano Occidente. 1994-2008
}

\author{
VICTORIA PICKETT S.*
}

\author{
Evaluation of the study of tuberculosis contacts in Western Public Health \\ Service of Santiago de Chile. 1994-2008
}

The evaluation of 15 years of study of TB contacts done in the western area of the Public Health Service of Santiago de Chile, 1994-2008, is presented. During this period an improvement on the coverage of this studies -even beyond the Ministry of Health aims- was observed. For this purpose the retrospective analysis of cases incorporated during the period was used. For definitions of healthy or diseased contact we followed the Manual of Tuberculosis of the National Tuberculosis Control Program of Chile. 9,344 intra-domicilliary and extra-domicilliary contacts were analized out of 2,129 index cases of bacillary $T B$ and index cases of children with TB. The coverage, morbility, diagnosis of TB and chemoprophylaxis carried out were evaluated. The study of contacts is probably the activity of case finding of the Program of Control of Tuberculosis most useful. In children under 15 years-old allows to find recent infections and treat them with chemoprophylaxis to avoid the development of new patients and the maintenance of the chain of transmission of the disease.

Key words: Tuberculosis contacts, Chilean tuberculosis control program, case finding, chemoprophylaxis.

\section{Resumen}

Se presenta la evaluación de 15 años del estudio de contactos de tuberculosis (TBC) del Servicio de Salud Metropolitano Occidente de la Región Metropolitana, correspondiente al período 1994-2008, en el que se observa una mejoría creciente de la cobertura de estos estudios, la que incluso superó la meta propuesta por el Ministerio de Salud. El método utilizado para esta investigación consistió en el análisis retrospectivo de los casos ingresados en el período. Para la definición de contacto sano o enfermo se tomó como referencia el Manual de Organización y Normas Técnicas y el Manual de Procedimientos para la Atención Primaria del Programa Nacional de Control de la Tuberculosis de Chile. Se analizó 9.344 contactos intra-domiciliarios y habituales extra-domiciliarios, que correspondieron a 2.129 casos índices de adultos con TBC pulmonar con bacteriología positiva y casos índices infantiles de TBC en todas sus formas. Se evaluó la cobertura, morbilidad, diagnóstico de casos TBC y quimioprofilaxis efectuadas. El estudio de contactos es probablemente la actividad de pesquisa tuberculosa focalizada de mayor rendimiento del Programa de Control. En el menor de 15 años de edad, este estudio permite pesquisar infecciones recientes, que tratadas adecuadamente con quimioprofilaxis, evitan la aparición de nuevos enfermos y la mantención de la cadena de transmisión de la enfermedad.

Palabras clave: Contactos de tuberculosis, control de la tuberculosis, pesquisa de casos, quimioprofilaxis.

\section{Introducción}

Contactos TBC son las personas que han estado expuestas al contagio con un enfermo de tuberculosis pulmonar con bacteriología positiva y que, por lo tanto, tienen más posibilidades de haberse infectado recientemente y de desarrollar la enfermedad. Constituyen uno de los grupos de más alto riesgo en tuberculosis, por lo que deben ser examinados con prioridad en un plazo máximo de 15 días después del diagnóstico del caso índice.

* Enfermera Asesora del Programa de Tuberculosis, Ministerio de Salud. Ex Enfermera Programa TBC, SSM Occidente. 
En Chile, entre 2 y $3 \%$ de los contactos examinados al momento del diagnóstico del caso índice enferman de tuberculosis, lo que representa un riesgo alrededor de 30 veces mayor al de la población general.

Desde el año 2005, la evaluación de los Estudios de Contactos, se incorporó a la evaluación operacional anual del Programa Nacional de Control de la Tuberculosis (PCT), posteriormente a las investigaciones operacionales realizadas en el Servicio de Salud Occidente el año 1994 y en la Región Metropolitana el año 1995, que demostraron bajas coberturas y alto porcentaje de morbilidad en esos contactos.

El objetivo de este estudio está dirigido a ofrecer en forma oportuna Diagnóstico, Tratamiento y Quimioprofilaxis, de acuerdo a las Normas del PCT a los contactos de casos índices contagiosos (TBC pulmonar con bacteriología positiva en mayores de 15 años) y a los casos índices enfermos (TBC en todas las formas, confirmadas y sin confirmar, en menores de 15 años de edad).

En el "Manual de Procedimientos para la Atención Primaria" del PCT se recomienda la meta de lograr $90 \%$ de estudios de contactos completos, ampliando este concepto y extendiéndolo no sólo a los contactos intra-domiciliarios, sino también a los habituales extra-domiciliarios. La responsabilidad del estudio y seguimiento del cumplimiento de los exámenes de contactos es de la enfermera de cada establecimiento, pero todo el equipo de salud, médicos y técnicos paramédicos deben revisar y reforzar las indicaciones.

Se presenta a continuación la evaluación del estudio de contactos TBC del Servicio Metropolitano Occidente de Santiago correspondiente al período 1994-2008, el que comprende a contactos de casos índices adultos de TBC pulmonar con bacteriología positiva y a casos índices infantiles de TBC en todas sus formas.

El método utilizado para este estudio consistió en el análisis retrospectivo de los casos ingresados en el período, mediante la evaluación de las tarjetas de contactos, respuestas de interconsultas y resultados de los exámenes practicados.

Se tomó como referencia el Manual de Procedimientos para la Atención Primaria y el Manual de Organización y Normas Técnicas del PCT vigentes para la definición de contacto sano o enfermo, considerando como estudio completo a los casos con el siguiente registro de resultados:

\section{- Contactos < 15 años:}

1. Revisión de cicatriz de BCG.

2. PPD.

3. Radiografía de tórax.
4. Estudio de Koch: directo y cultivo (2 muestras) a los sintomáticos respiratorios.

5. Decisión diagnóstica

\section{- Contactos > de 15 años:}

1. Radiografía de tórax.

2. Estudio de Koch: directo y cultivo (2 muestras) a los sintomático respiratorios.

3 Decisión diagnóstica.

Los datos que se presentan reafirman el alto riesgo de enfermar que presentan los contactos de enfermos tuberculosos confirmados por bacteriología positiva y la importancia que tiene el examen de sus contactos, ya que permite el diagnóstico oportuno de formas incluso muy graves y la aplicación de quimioprofilaxis en los menores de 15 años.

Se destaca el trabajo realizado por los equipos de enfermería de los establecimientos de la Red Occidente de Santiago, lo que permitió superar la meta propuesta por el Ministerio de Salud (MINSAL) (Figura 1).

El total de contactos censados en el período 1994-2008, en los establecimientos de la Atención Primaria y Secundaria de la Red Occidente de Santiago, correspondió a 9.344 contactos y a 2.129 casos índices de adultos con TBC pulmonar con bacteriología positiva y casos índices infantiles de tuberculosis en todas sus formas. La proporción de contactos por caso índice en el S. Occidente, corresponde a 4,4 contactos por cada caso índice.

Este análisis incluye no sólo a los contactos intra-domiciliarios, sino también a los habituales extra-domiciliarios (Figura 2).

En el año 1994, se observa baja cobertura de estudios completos $(21,4 \%$ en contactos menores y mayores de 15 años). Desde el año 1995 hay una creciente mejoría, llegando a superar la meta MINSAL (90\%) el año 2008.

Las estrategias que se utilizaron para incentivar esta actividad a partir del año 1994 fueron: talleres de capacitación para enfermeras, cursos de capacitación para equipos locales, capacitación de pediatras, ampliación de la red de atención de contactos menores de 15 años en los hospitales de Melipilla, Talagante y Peñaflor, reuniones de evaluación periódicas y difusión de resultados anuales (Figura 3).

La cobertura del estudio de los contactos en menores de 15 años en el año de inicio de la evaluación (15\%), fue inferior a la cobertura del total de ese período (21\%). Desde el año 1996 hasta el 2000, se observan mejores coberturas en el grupo de contactos menores de 15 años en relación al total de contactos. 


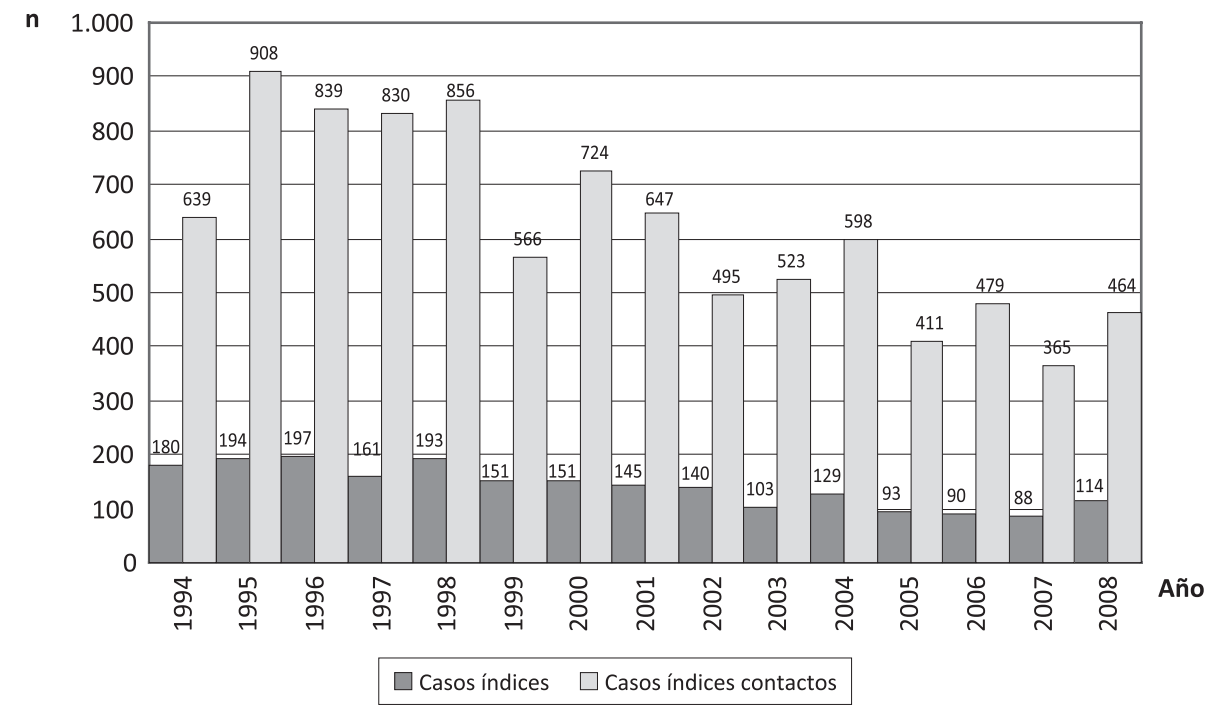

Figura 1. Casos índices y contactos censados.

Figura 2. Cobertura de estudios de contactos completos.

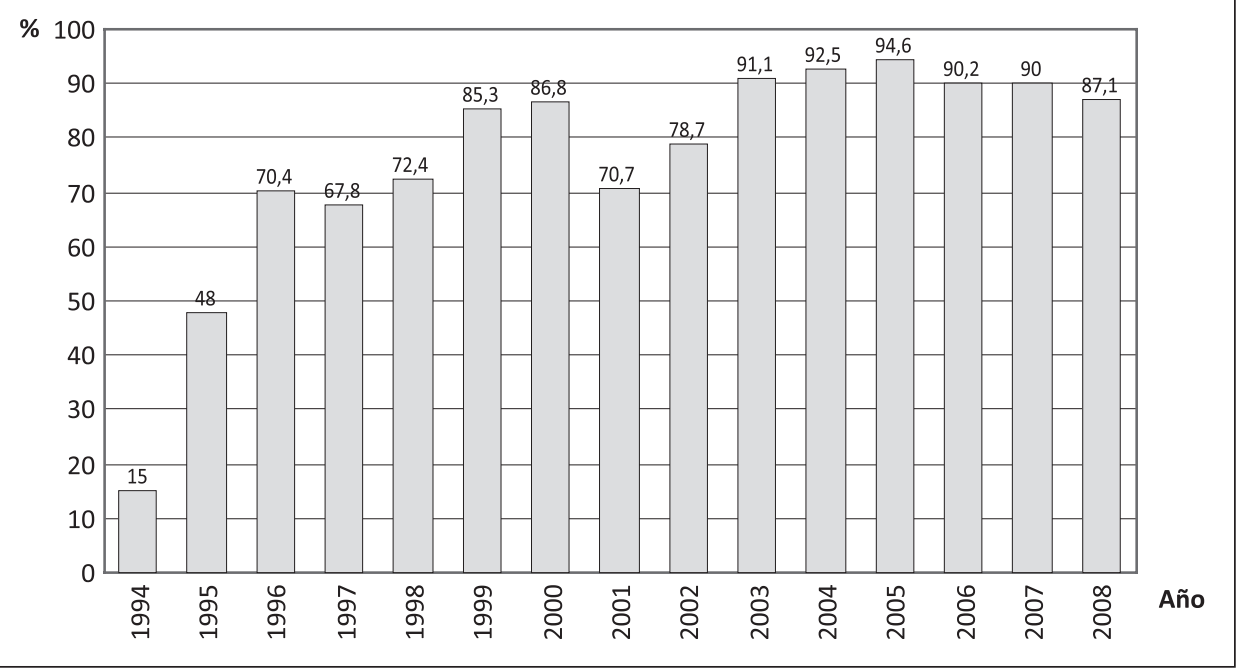

Figura 3. Cobertura de estudios de contactos completos en menores de 15 años. 

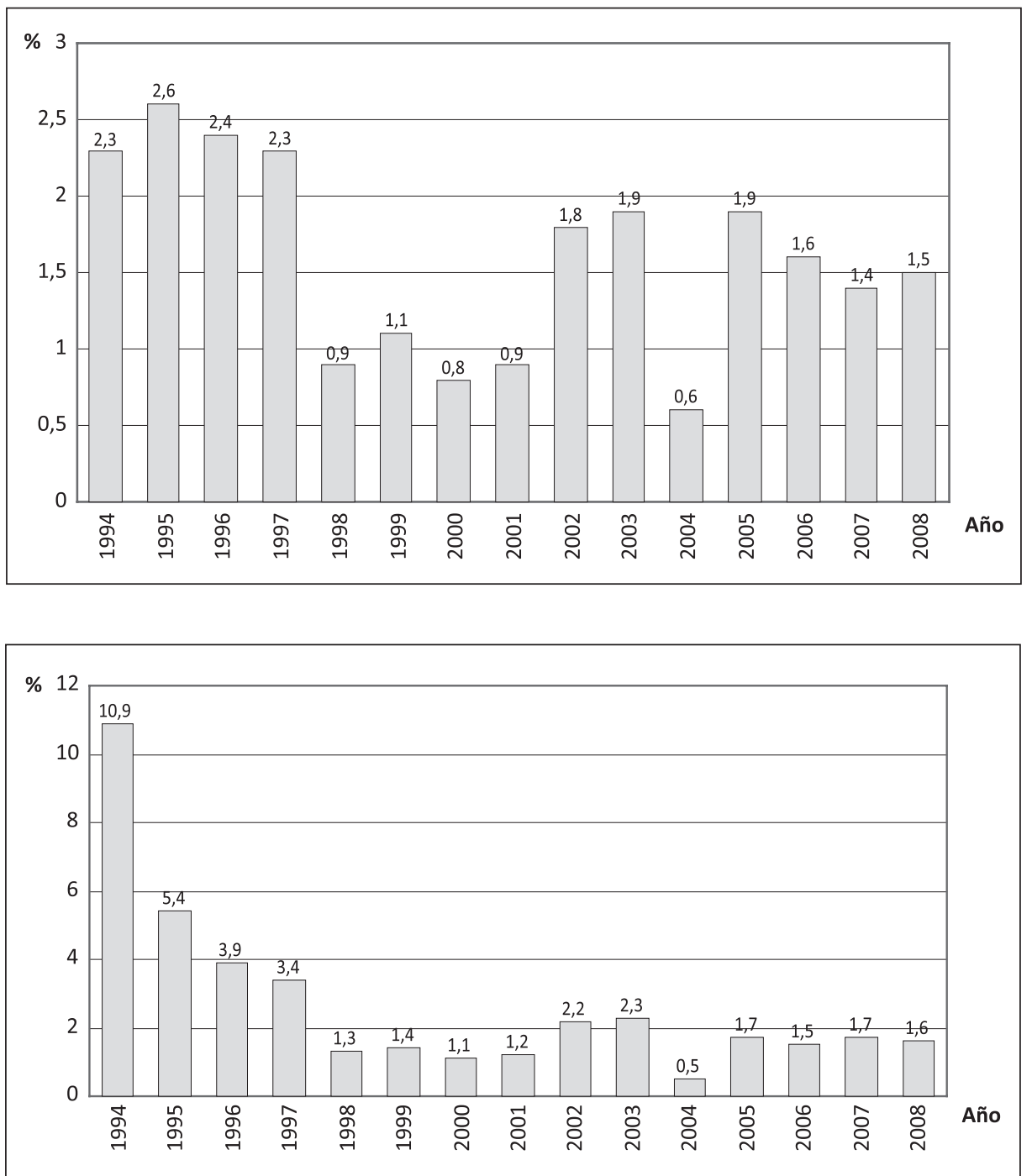

Figura 4. Morbilidad por TBC en contactos censados.
Figura 5. Morbilidad por TBC en contactos estudiados.
Entre los años 2003-2007 se logró superar la meta de cobertura de $90 \%$ del MINSAL entre los contactos menores de 15 años. Sin embargo, el año 2008 esta cobertura bajó a 87,1\%, siendo inferior a la lograda en el mismo año para el total de contactos $(93,1 \%)$ (Figura 3).

Al mejorar la cobertura de los estudios completos, el porcentaje de morbilidad bajó de 10,9\% el año 1994 a 1,5\% el año 2008 (Figuras 4 y 5).

El porcentaje de morbilidad TBC en todo el período evaluado, entre los contactos con estudios completos $(2,2 \%)$ fue mayor que entre los contactos censados $(1,6 \%)$ y comparable a las cifras del MINSAL (2 a 3\%), lo que demuestra el elevado riesgo de enfermar a que están expuestos (Tabla 1).

La morbilidad por TBC entre los contactos estudiados en el período evaluado, correspondió al diagnóstico de 150 casos de TBC, 39 casos en contactos menores de 15 años y 111 casos en mayores de 15 años (Tabla 2).

En el período de la evaluación (1994-2008) se diagnosticaron 124 casos de TBC pulmonar ( $82,7 \%$ del total de diagnósticos de tuberculosis) entre los contactos estudiados y 11 casos $(6,7 \%)$ de TBC extrapulmonar (6 pleural, 4 ganglionar y 1 miliar).

Los años 1994 y 1995 se diagnosticaron 6 casos de TBC inaparente, los que de acuerdo a las Normas de TBC Infantil en uso en 1994, correspondían a virajes tuberculínicos recientes y fueron tratados como tuberculosis activas. A contar de 1995 estos casos se consideran como infecciones recientes y tienen indicación de quimioprofilaxis (Tablas 3 y 4).

En el Servicio Occidente de Santiago a contar del año 1995, se cumple con la administración de quimioprofilaxis con isoniacida a los contactos 
TBC menores de 15 años, de acuerdo a las normas vigentes del PCT. Es importante destacar que la administración de la quimioprofilaxis a los 1.493

Tabla 1. Morbilidad por TBC en contactos menores y mayores de 15 años

\begin{tabular}{|cccc|}
\hline Años & $\begin{array}{c}\mathbf{n} \text { casos TBC } \\
<\mathbf{1 5} \text { años }\end{array}$ & $\begin{array}{c}\mathbf{n} \text { casos TBC } \\
>\mathbf{1 5} \text { años }\end{array}$ & $\begin{array}{c}\text { Total de } \\
\text { casos TBC }\end{array}$ \\
\hline 1994 & 3 & 12 & 15 \\
1995 & 8 & 16 & 24 \\
1996 & 7 & 13 & 20 \\
1997 & 7 & 12 & 19 \\
1998 & 0 & 8 & 8 \\
1999 & 0 & 6 & 6 \\
2000 & 1 & 5 & 6 \\
2001 & 2 & 4 & 6 \\
\hline 2002 & 2 & 7 & 9 \\
2003 & 3 & 7 & 10 \\
\hline 2004 & 2 & 1 & 3 \\
\hline 2005 & 0 & 6 & 6 \\
\hline 2006 & 0 & 6 & 6 \\
\hline 2007 & 2 & 3 & 5 \\
\hline 2008 & 2 & 5 & 7 \\
\hline Total & 39 & 111 & 150 \\
\hline
\end{tabular}

menores de 15 años durante este período, permitió cumplir con el objetivo de prevenir el desarrollo de la enfermedad en estos niños (Tabla 5).

De acuerdo a los resultados obtenidos en esta evaluación se concluye que en el Servicio de Salud Metropolitano Occidente se han obtenido los siguientes logros:

- Cumplimiento de las Normas del Estudio de Contactos.

- Mejoría creciente de la cobertura de Estudios Completos.

- Cobertura del año 2008 superó la meta MINSAL.

- Porcentaje de Morbilidad de 2,2\% es similar a cifra de la Norma Técnica del PCT.

- Diagnóstico de 150 Casos de TBC entre los contactos estudiados.

- Confirmación bacteriológica en $62 \%$ de los diagnósticos de TBC.

- Cumplimiento de la norma de quimioprofilaxis en el 53,3\% (1.493 niños).

Es recomendable mantener el registro completo de los resultados de los exámenes de los contactos en las tarjetas de tratamiento.

Se destaca el trabajo del equipo de enfermería de los establecimientos.

Esta evaluación confirma el alto riesgo de ser infectados y de enfermar que tienen las personas
Tabla 2. Formas clinicas de TBC en el total de los contactos

\begin{tabular}{|rcrcccr|}
\hline Años & $\begin{array}{c}\text { Complejo } \\
\text { primario } \\
\text { inaparente }\end{array}$ & Pulmonar & Miliar & Pleural & Ganglionar & Total \\
\hline 1994 & 3 & 12 & 0 & 0 & 0 & 15 \\
\hline 1995 & 5 & 17 & 1 & 0 & 1 & 24 \\
\hline 1996 & 1 & 18 & 0 & 1 & 0 & 20 \\
\hline 1997 & 1 & 16 & 0 & 0 & 2 & 19 \\
\hline 1998 & 0 & 8 & 0 & 0 & 0 & 8 \\
\hline 1999 & 0 & 6 & 0 & 0 & 0 & 6 \\
\hline 2000 & 0 & 6 & 0 & 0 & 0 & 6 \\
\hline 2001 & 0 & 6 & 0 & 0 & 0 & 6 \\
\hline 2002 & 2 & 6 & 0 & 1 & 0 & 9 \\
\hline 2003 & 1 & 7 & 0 & 1 & 1 & 10 \\
\hline 2004 & 0 & 2 & 0 & 1 & 0 & 3 \\
\hline 2005 & 0 & 6 & 0 & 0 & 0 & 6 \\
\hline 2006 & 0 & 6 & 0 & 0 & 0 & 6 \\
\hline 2007 & 1 & 3 & 0 & 1 & 0 & 5 \\
\hline 2008 & 1 & 5 & 0 & 1 & 0 & 7 \\
\hline Total & 15 & 124 & 1 & 6 & 4 & 150 \\
\hline
\end{tabular}

Tabla 3. Número de quimioprofilaxis en contactos menores de 15 años

\begin{tabular}{|cc|}
\hline Años & Quimioprofilaxis \\
\hline 1994 & 0 \\
1995 & 61 \\
\hline 1996 & 154 \\
1997 & 131 \\
\hline 1998 & 157 \\
1999 & 122 \\
\hline 2000 & 119 \\
2001 & 83 \\
\hline 2002 & 78 \\
\hline 2003 & 111 \\
\hline 2004 & 124 \\
2005 & 96 \\
\hline 2006 & 96 \\
\hline 2007 & 79 \\
\hline 2008 & 82 \\
\hline Total & 1.493 \\
\hline
\end{tabular}


Tabla 4. Porcentaje de quimioprofilaxis en contactos menores de 15 años en relación al total de contactos menores de 15 años censados

$\begin{array}{ccc}\text { Contactos }<\mathbf{1 5} \text { años (n) } & \text { Quimioprofilaxis }<\mathbf{1 5} \text { años (n) } & \text { Quimioprofilaxis }<\mathbf{1 5} \text { años (\%) } \\ 2.803 & 1.493 & 53,3\end{array}$

Tabla 5. Resumen de la evaluación del estudio de contactos en los menores de 15 años (1994-2008)

\begin{tabular}{|lrc|}
\hline Indicador & & Total 1994-2008 \\
Contactos censados & (n) & 9.344 \\
Casos índices & (n) & 2.129 \\
Contactos Estudiados & (n) & 6.655 \\
Contactos por caso índice & $(\%)$ & 4,4 \\
Contactos con tuberculosis & (n) & 150 \\
Morbilidad TBC en contactos estudiados & $(\%)$ & 2,2 \\
Contactos con quimioprofilaxis & $(\mathrm{n} / \%)$ & $1.493 / 53,3$ \\
\hline
\end{tabular}

que son contactos de enfermos con tuberculosis pulmonar con baciloscopia positiva.

\section{Bibliografía}

1.- MINSAL. Manual de Organización y Normas Técnicas Programa Nacional de Control de la Tuberculosis. 2005.

2.- MINSAL. Manual de Procedimientos para la Atención Primaria Programa Nacional de Control de la Tuberculosis. 2006.

3.- FARGA V, CAMINERO J A. Tuberculosis. $3^{\circ}$ edición.
Editorial Mediterráneo. Santiago de Chile. 2011.

4.- PICKETT V, POBLETE R, TORRES Z, O’ RYAN L, FLORES I. Evaluación del estudio de los Contactos de Tuberculosis en la Región Metropolitana. Rev Chil Enf Respir 1995; 11: 232 (Resumen CL-38).

5.- PICKETT V, CHAPARRO L, YÁÑEZ A. Evaluación del estudio de los Contactos de casos de tuberculosis pulmonar confirmados bacteriológicamente. Serv. Salud Metropolitano Occidente 1994 y 1995. Rev Chil Enf Respir 1996, 12: 128-30.

6.- FARGA V. Grupos de alto riesgo en tuberculosis. Estudio de contactos. Rev Chil Enf Respir 1996; 12: 126-7.

Correspondencia a:

Sra. Victoria Pickett S.

Enfermera asesora,

Programa de Control de la Tuberculosis

Ministerio de Salud, Santiago, Chile.

E-mail: vpicketts@hotmail.com 